\title{
Development of Correlation for Thermophysical Properties of Supercritical Oxygen to Be Used in SMES
}

\author{
Aal Arif Sarkar, Abhinav Kumar, Preeti Rao Usurumarti, and Raja Sekhar Dondapati
}

\begin{abstract}
One of the most promising applications in High Temperature superconductor (HTS) is Superconducting Magnetic Energy Storage (SMES). At present, electrical storage systems reports $50-60 \%$ losses due to traditional use of conventional conductors. In order to overcome such challenges, HTS with efficient cooling must be employed in power applications. In the present work, a novel cooling concept with supercritical oxygen (SCO) has been proposed to maintain the superconducting state for wide range of applications. Moreover, the thermophysical properties such as viscosity, density, thermal conductivity and specific heat have been studied over a wide range of temperatures $(154.58 \mathrm{~K}-204.58 \mathrm{~K})$ and critical pressure 50.43bar. Further, the development of correlations for SCO above the critical temperature $\left(T_{c}+50 \mathrm{~K}\right)$, and at critical pressure $\left(P_{c}+1\right.$ bar $)$ have been analyzed. The developed correlations can be used in predicting the performance of SMES power devices by prior modeling and simulations at various operating conditions.
\end{abstract}

Index Terms - Correlation, SMES, supercritical oxygen, and thermophysical properties.

\section{INTRODUCTION}

Nowadays, renewable energy sources (RESs) have recently become of particular interest, both in terms of intrinsically alternating resources and stability maintenance in electrical power systems [1]-[3]. Also, RESs power has been growing due to its immense availability and its truncated effect to the environment. However, these RESs power potential repeatedly changes and are barely predictable due to change in the environmental conditions. This can lead to undecorated complications due to severe fluctuations of tie-line power flow [4]. To overcome this problem, superconducting magnetic energy storage (SMES) can be utilized as an effective device with the ability to possess high storage efficiency, rapid response (within few milliseconds) and high cyclability, but only for short periods of time [5]. Essentially a SMES device comprises of three foundations: a magnet, a cryostat, and a connection system to the load [6]. The general components of the proposed SMES unit is shown in Fig. 1. In this study we focus mainly on the cryostat because of its emphasis on cooling the superconducting system. SMES are cryogenic devices, whose temperatures need to be kept sufficiently low to ensure non-dissipative operation of the

Manuscript received March 29, 2015; revised July 21, 2015

Aal Arif Sarkar and Raja Sekhar Dondapati are with Lovely Professional University, India (e-mail: aalarif63@gmail.com, rajasekhar.iitkgp@gmail.com).

Preeti Rao Usurumarti is with P.V.K. Institute of Technology, Andhra Pradesh, India (e-mail: preeti.iitkgp@gmail.com).

Abhinav Kumar is with St. Soldier Institute of Engineering and Technology, Jalandhar, India (e-mail: abradhe131213@ gmail.com). high temperature superconducting (HTS) wires. The temperature is basically enacted by the cold source which is used and generally fixed before the SMES coil design. The isolation of coil cannot be done effortlessly from the outside because there are heat losses by radiation from the cryostat external surface, by conduction through the mechanical support of the coil, the cryostat and the current leads to connect it electrically. Moreover, current leads and networks between the superconducting wires are creating losses by Joule effect, while the coil winding itself generates heat during operation, when the conductor is close from its critical current. In consequence, a thermal system must be designed to ensure that the coil temperature is low enough to allow safe operation, with a reasonably homogenous and stable temperature. The cooling system has therefore to absorb this heat flux otherwise the temperature would slowly increase, which would progressively increase the heat dissipation and trigger a slow thermal runaway. Energy is therefore being dissipated in small volumes, also known as hot spots, where temperature rises rapidly. Thermal expansion in the hot spots might cause high mechanical stresses and possibly deformations to which high temperature superconducting (HTS) materials performances are highly vulnerable.

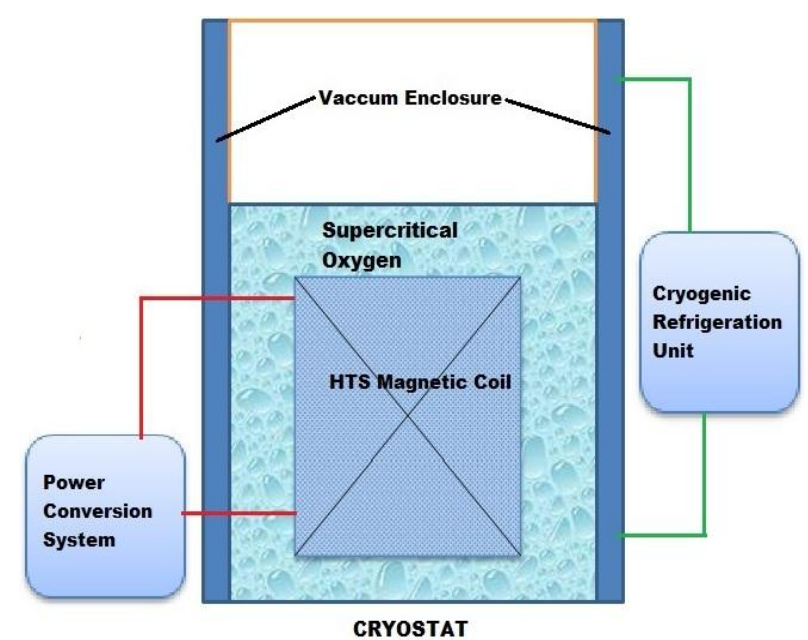

Fig. 1. General components of the proposed SMES unit.

Even if the conductor sustains the stress, when the temperature goes higher than the critical value the conductors linking might starts melting and the cryogens concentration in the superconducting material may get altered, which will ultimately cause the loss of superconducting properties. Therefore it is essential to have a good cryogenic fluid in the cryostat to bound the hotspots maximum temperature and the temperature gradients along the superconducting coil which requires an active protection [7]-[9]. Based on these facts, the aim of current work is to obtain novel cryogenic supercritical 
oxygen (SCO) fluid to be used in cryostat for cooling the high temperature superconducting (HTS) magnetic coil and bound the hotspots. Also we develop the explicit correlations for estimating the thermophysical properties of supercritical oxygen (SCO). The properties being a function of temperature and having wide range of applications is valid for temperature range from 155.78 to $204.58 \mathrm{~K}$ at pressure $50.53 \mathrm{bar}$. The main aim is to verify the actual performance of the cooling system of the SMES using SCO, which will enable us to effectively increase the transient and dynamic stability of the power system.

\section{THERMOPHYSICAL PROPERTIES OF SCO}

The need to bind the thermal hotspots generated due to heating in the coil and to enhance the heat transfer process, the analysis for specific properties of SCO to be used in SMES has to be carried out. The emphasis of analysis is laid on the assumption of SCO having spherical molecules. Also considering the ability of SCO to conduct the heat generated in coil and to quantify the heat content of a fluid or change in temperature of a fluid for an amount of heat generated, the specific gravity of fluid and mass transport property of SCO enabled us to take advantage of a correct combination of the properties which is needed to be analyzed for the application of SCO in SMES. Therefore, for the novel SCO cryogen, density, specific heat, viscosity and thermal conductivity are the significant thermophysical properties which are being studied here with respect to variation in temperatures and pressures. Fig. 3 illustrates the effect of temperature on viscosity at different pressures. It can be observed that with the increase in pressure, huge increase in the viscosity may be obtained in supercritical region. However, in the temperature range of $154.58 \mathrm{~K}<T<181.48 \mathrm{~K}$, an initial decrease in viscosity is identified. In addition, a small increase in viscosity is observed at the end of the temperature range $181.48 \mathrm{~K}<T<204.58 \mathrm{~K}$ due to the increase in density as can be seen from Fig. 3. Also, Fig. 2 shows the variation of density with temperature. In the pressure range of $50.43<P<51.33 \mathrm{bar}$, exciting rise in density is observed. However, within the temperature of $154.58 \mathrm{~K}<T<204.58 \mathrm{~K}$ density is regularly decreasing. Fig. 4 shows the variation of specific heat with temperature. It can be observed that with increase in pressure from 50.43bar to 50.53bar drastic decrease in specific heat. In addition, a constant fall in specific heat with further increase in pressure from 50.63bar to 51.36bar. However, effect of temperature on specific heat results in development of pseudo-critical points in the temperature range of $154.58 \mathrm{~K}<T<155.08 \mathrm{~K}$ and pressure range of $50.53 \mathrm{bar}$ to 51.33 bar. Furthermore, a linear drop in specific heat values is observed in the temperature range $155.08 \mathrm{~K}<T<204.58 \mathrm{~K}$. Fig. 5 relates thermal conductivity as function of temperature at different pressures. The graph demonstrates significant decrease in thermal conductivity during $50.43<P<50.53$ bar. Subsequently gradual fall is observed with further increase in pressure. However, by raise in the temperature, curves reveals uniformly diminishing values of thermal conductivity in the temperature range of $154.58 \mathrm{~K}<T<189.78 \mathrm{~K}$ and linear increase in the values in the temperature range of $189.78 \mathrm{~K}<T<204.58 \mathrm{~K}$.

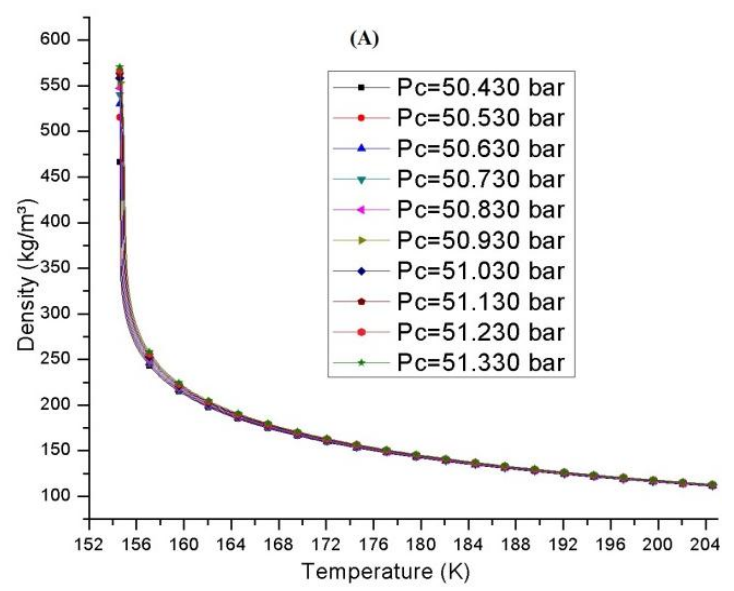

Fig. 2. Density at varying temperature and pressure.

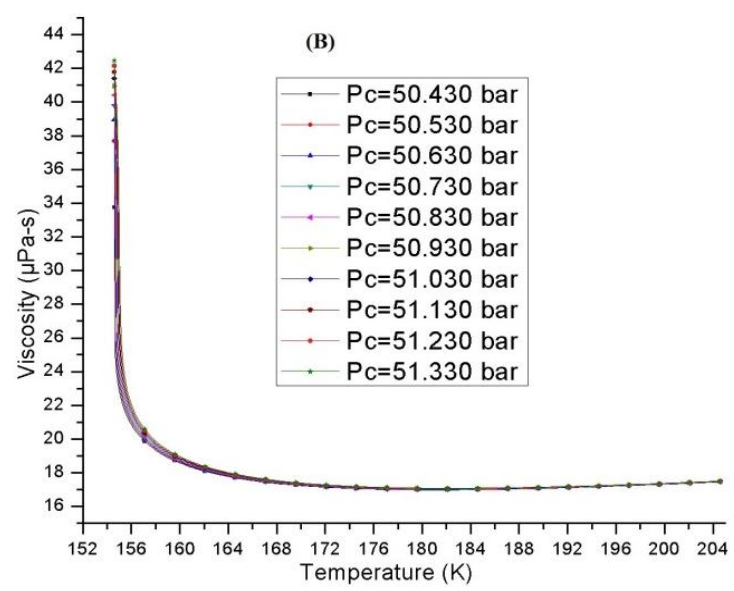

Fig. 3. Viscosity at varying temperature and pressure.

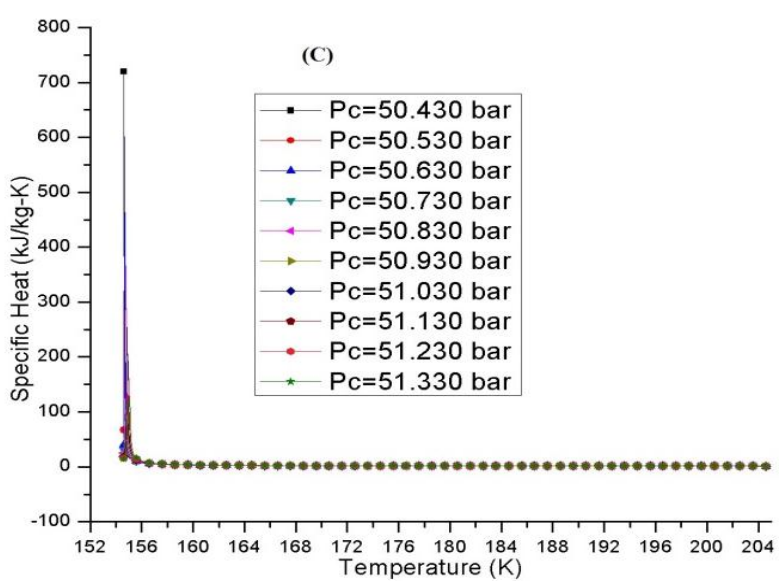

Fig. 4. Specific heat at varying temperature and pressure.

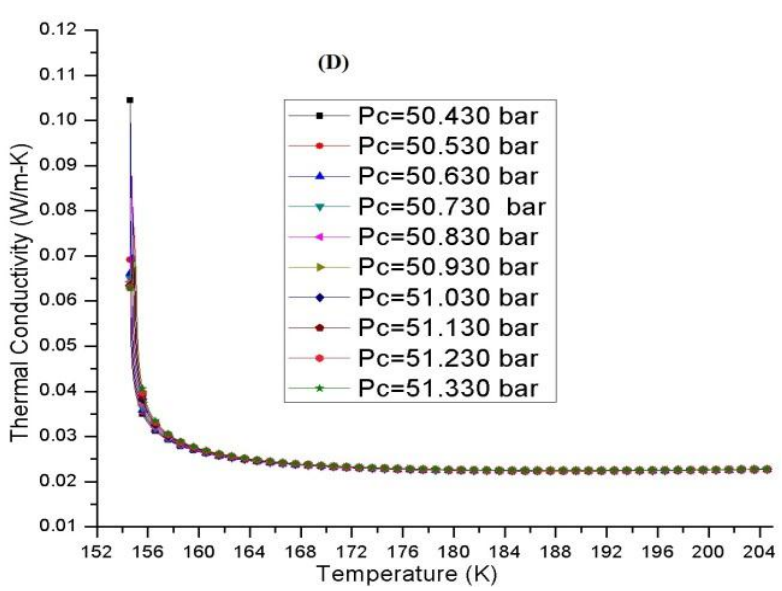

Fig. 5. Thermal conductivity at varying temperature and pressure. 
From the above study of thermophysical properties, it is observed that supercritical fluid i.e., SCO exhibit desirable intermediate properties of both gas and liquid phases [10]-[15]. This may be due to the fact that when oxygen is heated until its specific critical temperature $(154.58 \mathrm{~K})$ in a closed system, which means at constant pressure (50.43bar), a dynamic equilibrium is generated. This equilibrium includes the same number of molecules coming out of liquid phase to gas phase by gaining energy and going in to liquid phase from gas phase by losing energy thus affecting its molar volume. These co-existence of liquid and vapor at the same pressure with different molar volumes [16] leads to infinite isothermal compressibility at the critical point given by Eq. (1).

$$
\left(\frac{\partial P}{\partial V}\right)_{T}=0 \text { and }\left(\frac{\partial^{2} P}{\partial^{2} V}\right)_{T}=0
$$

This fluctuation in values results in the large inter-molecular distance, which is an indication of incipient instability near the critical points $(154.58 \mathrm{~K}$ and $154.68 \mathrm{~K})$ resulting in drastic drop in values of density, viscosity, specific heat and thermal conductivity of SCO.

However, due to this infinite compressibility factor, the increase in pressure at isotherms results in decreasing in intermolecular distance resulting in increase in the property values of density, viscosity, specific heat and thermal conductivity of SCO. This behavior depicts the property of $\mathrm{SCO}$ as a vapor. Moreover, at constant isochoric conditions when the temperature is increased, the specific gravity and isobaric heat capacity (degree of freedom) of SCO molecules also begins to increase in this region. As a result the diverging expansion coefficient of SCO molecules increases resulting in a smooth and gradual decrease in the property values. Thus, abruptly the viscosity, density, specific heat and thermal conductivity ascends from vapor-like to liquid-like values with variation in temperature.

\section{DEVELOPMENT OF CORRELATION}

Correlations have been developed in order to evaluate the variation of thermophysical properties with respect to temperature $\left(T_{C}+50 \mathrm{~K}\right)$ at critical pressure. These correlations were developed at critical pressure (50.43 bar) with the assumption that there is no significant change in the properties beyond $T_{C}+50 \mathrm{~K}$ in the supercritical region. Further, the developed correlations may be useful in thermal modeling of SMES. Table I predicts that thermophysical properties of SCO at critical temperature and pressure. Table II shows various correlations with their correlation coefficient values. These new correlation expressions were chosen of rational type because of its simplicity and less number of correlation coefficients. Moreover, it explains the relationship between dependent and independent variables. The difference between the magnitude of thermophysical properties at critical temperature and critical pressure is significantly larger than those above the critical temperature. In this event, critical temperature $\left(T_{C}=154.58 \mathrm{~K}\right)$ and $154.68 \mathrm{~K}$ of SCO was not considered for fitting at critical pressure $(P c=50.43 \mathrm{bar})$. A Total of 500 data points for each property has been taken from NIST [17] and analyzed.
TABLE I: THERMOPHYSICAL PROPERTIES AT CRITICAL TEMPERATURE $(T C=154.58 \mathrm{~K})$ AND PRESSURE $(P C=50.430 \mathrm{BAR})$

\begin{tabular}{l||llll}
\hline \hline \multirow{2}{*}{$\begin{array}{l}\text { Critical } \\
\text { Temperature } \\
(K)\end{array}$} & \multicolumn{4}{c}{ Thermophysical Properties } \\
\cline { 2 - 5 } & $\begin{array}{llll}\text { Density } \\
\left(\mathrm{kg} / \mathrm{m}^{3}\right)\end{array}$ & $\begin{array}{l}\text { Viscosity } \\
(\mu \mathrm{Pa}-\mathrm{s})\end{array}$ & $\begin{array}{l}\text { Thermal } \\
\text { conductivity } \\
(\mathrm{W} / \mathrm{m}-\mathrm{K})\end{array}$ & $\begin{array}{l}\text { Specific } \\
(\mathrm{kJ} / \mathrm{kg}-\mathrm{K})\end{array}$ \\
154.58 & 466.41 & 33.759 & 0.10442 & 719.48 \\
\hline \hline
\end{tabular}

\section{RESUlTS AND DISCUSSIONS}

To establish the accuracy of fitted model, statistical parameters such as Arithmetic Average of the Absolute Values of the Relative Errors (AARE\%) and Sum of Absolute of Residual (SAR) have been utilized. Small values of these parameters refer to reliable correlation. The Arithmetic Average of the Absolute Values of the Relative Errors (AARE \%) is defined in Eq. (2) [18]-[21].

$$
A A R E \%=\frac{100}{N} \sum^{N}\left(\left|\frac{\zeta^{\mathrm{exp}}-\zeta^{\mathrm{cal}}}{\zeta^{\exp }}\right|\right)
$$

Fig. 6 shows the AARE\% versus temperature for all the thermophysical properties of SCO at critical pressure. Another such parameter is the Sum of Absolute of Residual (SAR) which is defined in Eq. (3) [18]-[21], which put forth the reliability of correlation for more intense data points.

$$
S A R=\sum^{N}\left|\zeta^{\mathrm{exp}}-\zeta^{\mathrm{cal}}\right|
$$

The Average Percent Relative Error (ARE \%) is defined in Eq. (4) [18], [19], [21], which gives a measure of the foregone conclusion of the correlation. A value of zero indicates a random of the measured values around the correlation.

$$
A R E \%=\frac{100}{N} \sum^{N}\left(\frac{\zeta^{\exp }-\zeta^{\mathrm{cal}}}{\zeta^{\exp }}\right)
$$

Fig. 7, shows Percent Relative Error (RE \%) as function of temperature which is defined in Eq. (5) [18]-[21] for each thermophysical properties.

$$
R E \%=100 \times\left(\frac{\zeta^{\exp }-\zeta^{\mathrm{cal}}}{\zeta^{\exp }}\right)
$$

It can be concluded from the Fig. 7 that, with increase in temperature relative error is decreasing for the correlation developed. It shows clearly the reliability of developed correlations for higher property values as a function of temperature. The AARE\%, ARE\% and SAR values of the correlation developed for SCO in comparison with the NIST values of every thermophysical properties is revealed in Table III. About the developed correlations, the AARE\% at supercritical region is 0.120698633 for density, 0.223487687 for viscosity, 0.201117284 for specific heat and 0.193192467 for thermal conductivity in comparison with the NIST data. 


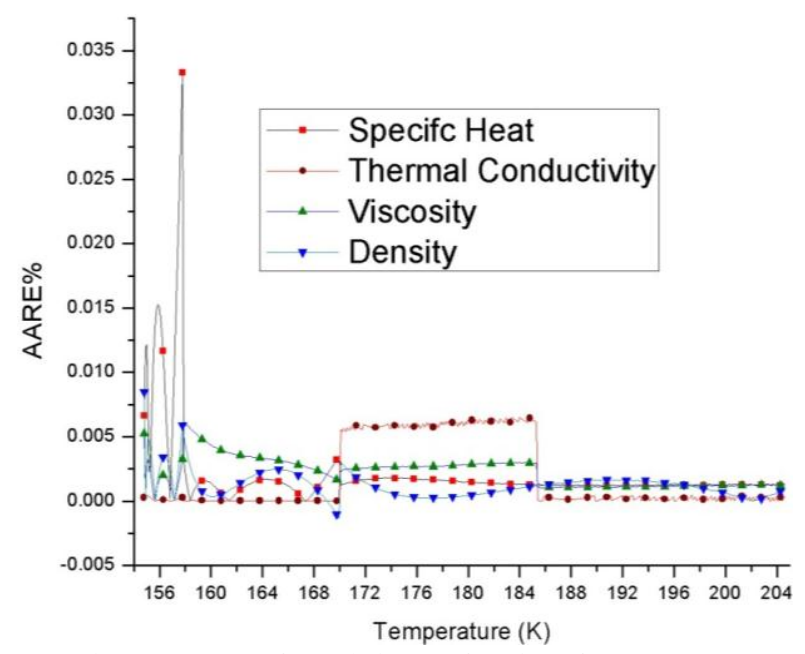

Fig. 6. AARE\% of correlations as function of temperature.

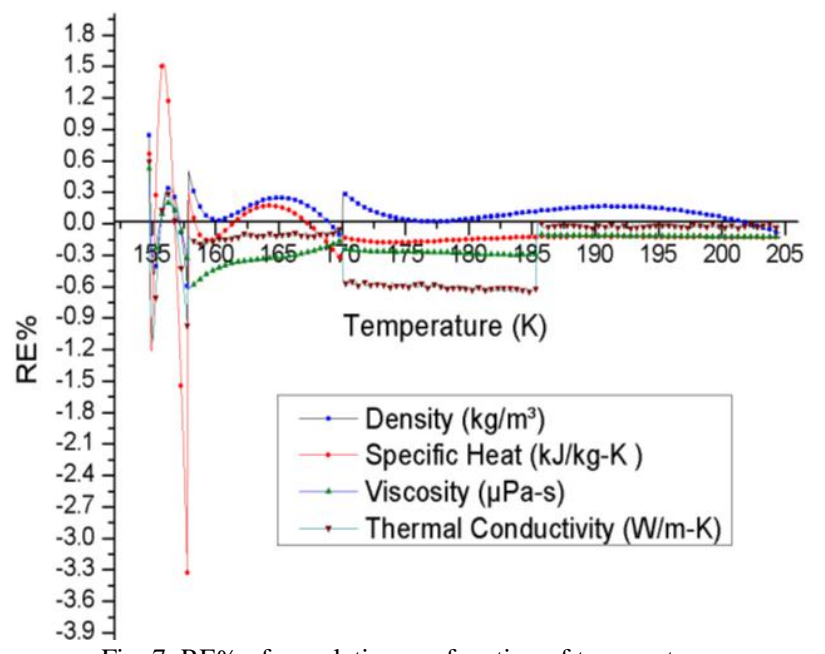

Fig. 7. RE\% of correlations as function of temperature.

TABLE II: DEVELOPED CORRELATIONS AND THEIR COEFFICIENTS FOR THERMOPHYSICAL PROPERTIES AT VARIOUS TEMPERATURE RANGE

\begin{tabular}{|c|c|c|c|c|}
\hline Properties & Temperature Range & \multicolumn{2}{|c|}{ Correlation and Correlation Coefficients } & $R^{2}$ Value \\
\hline \multirow{3}{*}{ Density } & $154.78 \mathrm{~K} \leq T \leq 157.78 \mathrm{~K}$ & $\rho(T)=\frac{\rho_{1}+\rho_{2} * T}{\rho_{0}+T}$ & $\rho_{0}=-153.20, \rho_{1}=-28833.47, \rho_{2}=189.54073$ & 0.99846 \\
\hline & $157.88 \mathrm{~K} \leq T \leq 169.98 \mathrm{~K}$ & $\rho(T)=\frac{\rho_{1}+\rho_{2} * T}{1+\rho_{0} * T}$ & $\rho_{0}=-0.00687, \rho_{1}=87.06, \rho_{2}=-0.67523$ & 0.99988 \\
\hline & $170.08 \mathrm{~K} \leq T \leq 204.58 \mathrm{~K}$ & $\rho(T)=\frac{\rho_{1}+\rho_{2} * T}{1+\rho_{0} * T}$ & $\rho_{0}=-0.00785, \rho_{1}=5.4524, \rho_{2}=-0.35549$ & 0.99997 \\
\hline \multirow{4}{*}{ Viscosity } & $154.78 \mathrm{~K} \leq T \leq 157.78 \mathrm{~K}$ & $\mu(T)=\frac{\mu_{1}+\mu_{2} * T}{\mu_{0}+T}$ & $\mu_{0}=-153.55, \mu_{1}=-2710.190, \mu_{2}=17.69972$ & 0.99864 \\
\hline & $157.88 \mathrm{~K} \leq T \leq 169.98 \mathrm{~K}$ & $\mu(T)=\frac{\mu_{1}+\mu_{2} * T}{1+\mu_{0} * T}$ & $\mu_{0}=-0.0066, \mu_{1}=15.92515, \mu_{2}=-0.10606$ & 0.99996 \\
\hline & $170.08 \mathrm{~K} \leq T \leq 185.28 \mathrm{~K}$ & $\mu(T)=\mu_{0}+\mu_{1} T+\mu_{2} T^{2}+\mu_{3} T^{3}$ & $\mu_{0}=209.80, \mu_{1}=-2.92, \mu_{2}=0.0146, \mu_{3}=-2.42 \mathrm{E}-5$ & 0.99903 \\
\hline & $185.38 \mathrm{~K} \leq T \leq 204.58 \mathrm{~K}$ & $\mu(T)=\mu_{0}+\mu_{1} T+\mu_{2} T^{2}+\mu_{3} T^{3}$ & $\mu_{0}=116.3, \mu_{1}=-1.46, \mu_{2}=0.00706, \mu_{3}=-1.11 \mathrm{E}-05$ & 0.99999 \\
\hline \multirow{3}{*}{$\begin{array}{l}\text { Specific } \\
\text { heat }\end{array}$} & $154.78 \mathrm{~K} \leq T \leq 157.78 \mathrm{~K}$ & $\mathrm{c}_{p}(T)=\frac{\mathrm{c}_{p 1}+\mathrm{c}_{p 2} * T}{\mathrm{c}_{p 0}+T}$ & $\mathrm{c}_{p 0}=-154.48, \mathrm{c}_{p 1}=-324.77, \mathrm{c}_{p 2}=2.15127$ & 0.99958 \\
\hline & $157.88 \mathrm{~K} \leq T \leq 169.98 \mathrm{~K}$ & $\mathrm{c}_{p}(T)=\frac{\mathrm{c}_{p 1}+\mathrm{c}_{p 2} * T}{\mathrm{c}_{p 0}+T}$ & $\mathrm{c}_{p 0}=-153.63, \mathrm{c}_{p 1}=-144.49, \mathrm{c}_{p 2}=1.0286$ & 0.99996 \\
\hline & $170.08 \mathrm{~K} \leq T \leq 204.58 \mathrm{~K}$ & $\mathrm{c}_{p}(T)=\frac{\mathrm{c}_{p 1}+\mathrm{c}_{p 2} * T}{1+\mathrm{c}_{p 0} * T}$ & $\mathrm{c}_{p 0}=-0.00661, \mathrm{c}_{p 1}=0.7434, \mathrm{c}_{p 2}=-0.00572$ & 1 \\
\hline \multirow{4}{*}{$\begin{array}{c}\text { Thermal } \\
\text { Conductivity }\end{array}$} & $154.78 \mathrm{~K} \leq T \leq 157.78 \mathrm{~K}$ & $\kappa(T)=\frac{\kappa_{1}+\kappa_{2} * T}{\kappa_{0}+T}$ & $\kappa_{0}=-154.00116, \kappa_{1}=-3.82176, \kappa_{2}=0.02492$ & 0.99824 \\
\hline & $157.88 \mathrm{~K} \leq T \leq 169.98 \mathrm{~K}$ & $\kappa(T)=\frac{\kappa_{1}+\kappa_{2} * T}{\kappa_{0}+T}$ & $\kappa_{0}=-151.82955, \kappa_{1}=-3.07204, \kappa_{2}=0.02056$ & 0.99999 \\
\hline & $170.08 \mathrm{~K} \leq T \leq 185.28 \mathrm{~K}$ & $\kappa(T)=\kappa_{0}+\kappa_{1} T+\kappa_{2} T^{2}+\kappa_{3} T^{3}$ & $\kappa_{0}=0.90, \kappa_{1}=-0.014, \kappa_{2}=7.4 \mathrm{E}-05, \kappa_{3}=-1.33 \mathrm{E}-07$ & 0.99998 \\
\hline & $185.38 \mathrm{~K} \leq T \leq 204.58 \mathrm{~K}$ & $\kappa(T)=\kappa_{0}+\kappa_{1} T+\kappa_{2} T^{2}+\kappa_{3} T^{3}$ & $\kappa_{0}=0.219, \kappa_{1}=-0.002, \kappa_{2}=1.3 \mathrm{E}-05, \kappa_{3}=-2.14 \mathrm{E}-08$ & 0.99999 \\
\hline
\end{tabular}

TABLE III: STATISTICAL VALUE FOR EACH THERMOPHYSICAL PROPERTIES AT VARYING TEMPERATURE AND PRESSURE

\begin{tabular}{c||ccc}
\hline \hline Properties & AARE\% & ARE\% & SAR \\
\hline \hline Density & 0.120698633 & 0.099056855 & 104.569232 \\
Viscosity & 0.223487687 & -0.213166932 & 19.70022503 \\
& & & \\
Specific heat & 0.201117284 & -0.105039577 & 3.879817087 \\
$\begin{array}{c}\text { Thermal } \\
\text { Conductivity }\end{array}$ & 0.193192467 & -0.235407374 & 0.029946928 \\
\hline \hline
\end{tabular}

Also the values of ARE\% for each thermophysical properties i.e., density, viscosity, specific heat and thermal conductivity are $0.099056855,0.2131669323,0.105039577$ and 0.23540734 respectively. It means that the correlations should be preferred over the NIST correlations. For this purpose 500 data points for each density, viscosity, specific heat and thermal conductivity have been selected. The temperature ranges of the data are $154.78-204.58 \mathrm{~K}$ at 50.43bar. Based on the obtained results in Fig. 6, Fig. 7 and Table III, the correlations developed along with correlation coefficients have good accuracy versus its simplicity.

\section{CONCLUSION}

An easy-to-use correlation has been proposed to accurately predict the density, specific heat, viscosity and thermal conductivity as a function of temperature in the present work. The main advantage of using this correlation is that it does not need big computations and large number of parameters but shows splendid concord to that of NIST values. The predictions showed admirable contract with those reported in the NIST. This novel correlation predicts the values of SCO 
in temperatures ranging between $154.78 \mathrm{~K}$ and $204.58 \mathrm{~K}$ for critical pressure 50.43bar. The proposed correlation has resulted in lower AARE\%, ARE\% and SAR which identifies it as a dependable approach for prior analysis of SMES power devices.

\section{ACKNOWLEDGMENT}

This work was supported in part by Lovely Professional University, Phagwara, India. Authors gratefully acknowledge the support of Applied Superconductivity Laboratory, IIT Kharagpur, India.

\section{REFERENCES}

[1] M. Tada, "Power control by superconducting magnetic energy storage for loading change compensation and power system stabilization in interconnected power system," IEEE Trans App. Superconductivity, vol. 5, no. 2, pp. 250-253, June 1995.

[2] M. Park et al., "HTS SMES application for the frequency stabilization of grid-connected wind power generation system," J. Supercond Nov. Magn., vol. 24, pp. 1007-1014, 2011.

[3] S. J. Cheng, "High temperature SMES for improving power system stabilities," Science in China Series E: Technological Sciences, vol. 50, no. 4, pp. 402-412, August 2007.

[4] S. Dechanupaprittha et al., "Stabilization of tie-line power flow by robust SMES controller for interconnected power system with wind farms," IEEE Trans. App. Superconductivity, vol. 17, no. 2, pp. 2365-2368, June 2007.

[5] Z. A. Baig and A.-R. Amoudi, "An analysis of smart grid attacks and counter measures," Journal of Communications, vol. 8, no. 8, pp. 473-479, August 2013.

[6] K. Fossheim and A. Sudbo, Superconductivity: Physics and Applications, John Wiley \& Sons Ltd, 2004, pp. 3-427.

[7] H. K. Yeom, Y. J. Hong, S. J. Park, T. B. Seo, K. C. Seong, and H. J. Kim, "Study of cryogenic conduction cooling systems for an HTS SMES," IEEE Transactions on Applied Superconductivity, vol. 17, no. 2, pp. 1955-1958, June 2007.

[8] V. Karasik, K. Dixon, C. Weber, B. Batchelder, G. Campbell, and P. Riberio, "SMES for power utility applications: A review of technical and cost considerations," IEEE Transactions on Applied Superconductivity, vol. 9, no. 2, pp. 541-546, June 1999.

[9] S. C. Tripathy, "Superconducting magnetic energy storage," Livewire, vol. 1, issue 1, pp. 11-14, 2005.

[10] R. B. Stewart, R. T. Jacobsen, and W. Wagner, "Thermodynamic properties of oxygen from the triple point to $300 \mathrm{~K}$ with pressures to 80MPa," J. Phys. Chem. Ref. Data, vol. 20, no. 5, pp. 917-1021,1991

[11] D. Beysens, J. Straub, and D. J. Turner, "Phase transitions and near-critical phenomena," in Fluid Sciences and Materials Science in Space European Space Agency, H. U. Walter, Ed. Paris: Cedex, pp. 221-256, 1987.

[12] S. B. Kiselev and V. D. Kulikov, "Thermodynamic and transport properties of fluids and fluid mixtures in the extended critical region," International Journal of Thermophysics, vol. 18, no. 5, pp. 1143-1182, 1997.
[13] I. L. Pioro, H. F. Khartabil1, and R. B. Duffey, "Heat transfer to supercritical fluids flowing in channels-empirical correlations (survey)," Nuclear Engineering and Design, vol. 230, pp. 69-91, 2004.

[14] F. Zhong and H. Meyer, "Heat transport in a pure fluid near the critical point: Steady state and relaxation dynamics," Journal of Low Temperature Physics, vol. 114, no. 1/2, pp. 231-255, 1999.

[15] X. D. Li, G. F. Xie, and R. S. Wang, "Experimental and numerical investigations of fluid flow and heat transfer in a cryogenic tank at loss of vacuum," Heat Mass Transfer, vol. 46, pp. 395-404, February 2010

[16] E. Kiran, "Supercritical fluids: Fundamental and applications," Series E: Applied Sciences, vol. 366.

[17] E. W. Lemmon, M. O. Mclinden, and M. L. Huber, "NIST standard reference database, physical and chemical properties division," Version 7.0 Beta, USA, 2012.

[18] E. Heidaryan and A. Jarrahian, "Modified redlich-Kwong equation of state for supercritical carbon dioxide," J. of Supercritical Fluids, vol. 81, pp. 92-98, 2013.

[19] A. Jarrahian and E. Heidaryan, "A novel correlation approach to estimate thermal conductivity of pure carbon dioxide in the supercritical region," J. of Supercritical Fluids, vol. 64, pp. 39-45, 2012.

[20] A. A. Amooey, "A simple correlation to predict thermal conductivity of supercritical carbon dioxide," J. of Supercritical Fluids, vol. 86, pp. $1-3,2014$

[21] A. A. Sarkar and R. S. Dondapati, "A novel approach to estimate the thermophysical properties of pure oxygen at the supercritical region to be used in SMES," in Proc. 2nd National Conference on Advancements in Simulation \& Experimental Techniques in Mechanical Engineering (NCASEme-2015), March 2015, pp. 67-75.

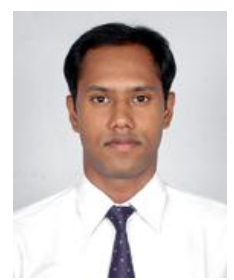

Aal Arif Sarkar was born in Assam, India on October 4,1990 . He is currently pursuing his master degree in thermal engineering at Lovely Professional University, India. His research activity includes cryogenics and applied superconductivity, particularly in superconducting magnetic energy storage system (SMES). He also has few international and national publications in the field of cryogenics.

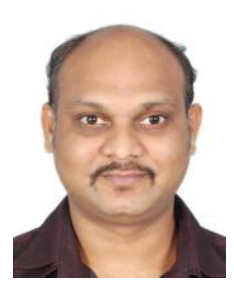

Raja Sekhar Dondapati graduated with the B.Tech degree in mechanical engineering from JNTU Kakinada and completed his post-graduation and received his M.Tech. degree from Sri Krishna Devaraya University Ananthapur. His PhD work from IIT Kharagpur includes cooling of superconducting magnets used for fusion grade magnets. He also holds positions in national and international bodies. His technological contributions to the society are numerous. Ministry of new and renewable energy of Govt. of India has appointed him as a coordinator of Renewable Energy Club (REC). He involved actively in the development of living standards of rural people. $\mathrm{He}$ also engaged in molding different societies to contribute to the society. 